\title{
EL SENTIDO DE LAS PRÁCTICAS EDUCATIVAS EN EDUCACIÓN AMBIENTAL
}

\author{
Raul Calixto-Flores \\ Universidad Pedagógica Nacional, México. rcalixto@upn.mx
}

\begin{abstract}
Resumen. Este capítulo tiene el objetivo de describir los resultados de una investigación sobre el sentido de las prácticas educativas en educación ambiental; la investigación realizada es cualitativa por medio de una encuesta y entrevistas a una muestra de 13 educadores ambientales, se destaca la conformación de las prácticas educativas orientadas a la generación de procesos de concientización, a través de estrategias y acciones que parten del reconocimiento de los problemas ambientales. Los sujetos de estudio desempeñan funciones diferentes, tienen diversas experiencias de vida, no cuentan con un nivel de formación académico común y viven en contextos diferentes, sin embargo, sus representaciones sociales sobre la educación ambiental son homogéneas, al poseer una identidad como educadores ambientales. En las conclusiones se confirman las implicaciones de la teoría de las representaciones sociales para comprender los conocimientos de sentido común que poseen los educadores ambientales y que le confieren un sentido a las prácticas educativas.
\end{abstract}

Palabras clave: Representaciones Sociales; Educación Ambiental; Investigación Cualitativa; Prácticas Educativas.

\section{THE MEANING OF EDUCATIONAL PRACTICES IN ENVIRONMENTAL EDUCATION}

\begin{abstract}
This chapter aims to describe the results of an investigation into the meaning of educational practices in environmental education; the research carried out is qualitative through a survey and interviews with a sample of 13 environmental educators, highlighting the formation of educational practices aimed at generating awareness processes, through strategies and actions based on the recognition of problems environmental. The subjects of study perform different functions, have different life experiences, do not have a common level of academic training and live in different contexts, however, their social representations of environmental education are homogeneous, having an identity as environmental educators. The conclusions confirm the implications of the theory of social representations to understand the common sense knowledge that environmental educators possess and that give meaning to educational practices.
\end{abstract}

Keywords: Social Representations; Environmental Education; Qualitative Research; Educational Practices.

\section{INTRODUCCIÓN}

En este capítulo se describen algunos hallazgos de una investigación se orientó hacia la interpretación del sentido de las prácticas en educación ambiental en una muestra de educadores ambientales, con base a la teoría de la representación social. En la delimitación del objeto de representación se toman en cuenta las condiciones que propone Díaz (1997): que el objeto de estudio efectivamente exista como objeto de representación; que los sujetos puedan expresar su representación social y que los instrumentos logren integrar los elementos que conforman a dicha representación. En trabajos previos (Calixto-Flores, GarcíaRuiz, \& Terrón-Amigón, 2015; Calixto-Flores \& Moreno-Elizalde, 2017; Calixto-Flores, 2018), con educadores ambientales, se ha confirmado la existencia de la educación ambiental como 
un objeto de representación en estudiantes y profesores de diversos niveles educativos. La investigación descrita en este documento, contempla entre sus objetivos identificar y analizar las representaciones sociales de los educadores ambientales sobre las prácticas educativas.

\section{LAS REPRESENTACIONES SOCIALES}

Las representaciones sociales tienen una naturaleza convencionalizadora y otra prescriptiva, con las cuales determinan y condicionan la experiencia de los sujetos, emergen en las interacciones cotidianas de cada grupo social. Moscovici (1979) refiere tres condiciones para que aparezcan: la dispersión de la información, la focalización del sujeto individual y colectivo y la presión a la inferencia del objeto socialmente definido. Estas condiciones hacen posible la determinación de la organización cognoscitiva de la representación de los sujetos. La representación social constituye una teoría que cuestiona el ideal del conocimiento científico, y revalora la importancia de estudiar el conocimiento de sentido común, como un conocimiento que da cuenta de algo real en su ausencia, organizado en estructuras (cognitivo-afectivas) que permiten darle sentido al entorno. Las representaciones sociales comprenden tres dimensiones información, campo de representación y actitudinal. En las representaciones sociales ocurren simultáneamente procesos interrelacionados de percepción, categorización y significación. La percepción determinada por la difusión y divulgación de los conocimientos científicos; la categorización de los distintos componentes que la constituyen; y la significación otorga el sentido a participar o compartir la representación. Las prácticas son producto de la vida cotidiana, surgen de ella como la manifestación del interior de los sujetos; a través de la comprensión de las prácticas, se puede entender cómo se conforman las relaciones entre los integrantes de diversos grupos.

En México, los resultados de los estudios de las representaciones sociales en educación, han contribuido a la comprensión de diversos hechos educativos; las primeras investigaciones documentadas que utilizaron la teoría de las representaciones sociales se remontan a la década de los 90 del siglo anterior.

Las investigaciones sobre las representaciones sociales se generan en diversas áreas educativas, como la formación docente, la práctica educativa, excelencia académica, calidad ambiental, entre otros. En la actualidad resulta difícil de precisar el número y diversas de temas que son investigados por medio de las representaciones sociales. Al respecto Cuevas y Mireles (2016), hacen una revisión de investigaciones que utilizan la teoría de la representación social, encontrando 141 trabajos, abordando diversos tópicos: prácticas 
docentes (16\%); política educativa (13\%); educación ambiental (11\%); instituciones escolares (8\%); formación e identidad profesional (7\%); sujetos (profesores, orientador, autoridades universitarias, niños) (6\%); formación docente (6\%); prácticas escolares de los estudiantes (6\%); trayectoria escolar (6\%); enseñanza de las matemáticas (6\%); el currículo (5\%); evaluación educativa en diferentes modalidades: institucional, docente y del aprendizaje (4\%); construcción social de la ciudadanía y los valores (4\%); y otros (8\%) en que se aborda temas emergentes como género, inclusión educativa y tecnologías de la información y la comunicación. Uno de los supuestos derivados de los estudios realizados, vincula la posibilidad de una mejor comprensión de prácticas en los diversos actores educativos a partir del reconocimiento de las representaciones sociales; y con ello emerge la posibilidad de realizar diferentes adecuaciones a las políticas y a las planeaciones escolares. Sautu (2007) refiere que las representaciones son referentes para que las personas organicen la interacción social, es decir constituyen un conocimiento práctico, con lo cual pueden dar sentido al mundo físico y social y habilitar la comunicación mediante códigos compartidos que permiten nombrar y clasificar aspectos del mundo social. Estas características hacen que la teoría de la representación social tenga un papel fundamental para comprender el sentido de las prácticas.

\section{RUTA METODOLÓGICA}

Para Watson-Gegeo (1982), la investigación cualitativa se caracteriza por realizar descripciones detalladas de situaciones, eventos, personas, interacciones y comportamientos que son observables. La investigación, que se describe en este capítulo, se dirige a la interpretación del sentido de las prácticas de un grupo de personas (educadores ambientales), por lo cual se delimita como un estudio descriptivo, de carácter interpretativo, orientada por el enfoque procesual de las representaciones sociales. Es un estudio descriptivo porque muestra las características de las representaciones de las prácticas educativas; es un estudio interpretativo porque interesa lo particular y lo contextual, de las representaciones, se comprende la realidad como dinámica y diversa.

La ruta metodológica tiene una vía inductiva-deductiva para el desarrollo de la investigación, se da la posibilidad de favorecer la valoración y apreciación de las personas involucradas en los procesos educativos. Se propone reconocer la valoración de las experiencias de los educadores ambientales, cuyo propósito es no solo incidir en las presencias y ausencias que se han producido alrededor de la cotidianeidad de la práctica. Es decir, en las 
representaciones de las prácticas se identifican las características, pero también las lógicas, con ausencias, problemas, retos, avances, constantes, entre otros aspectos. En esta investigación se empleó la encuesta y la entrevista semiestructurada, cada una permiten identificar los diversos componentes de las representaciones sobre las prácticas educativas. En este escrito solo se presentan los resultados de la entrevista, priorizando el análisis cualitativo de las expresiones textuales de los educadores ambientales.

La entrevista se realizó con base a guiones semiestructurados para identificar y caracterizar el sentido de las prácticas, se registraron y transcribieron los relatos de los educadores, a fin de reconstruir sus experiencias en torno a la educación ambiental. Las preguntas planteadas, comprendieron las tres dimensiones de las representaciones: información, campo de representación y actitudinal. Algunas de las preguntas planteadas de la dimensión de información son:

- ¿Cuáles materiales considera básicos conocer en el campo de la educación ambiental?

- ¿Cuáles son los aspectos que le resultan relevantes en la práctica en educación ambiental?

De la dimensión campo de representación:

- ¿Qué imagen le viene a la mente al escuchar educación ambiental?

- ¿Para qué realizar educación ambiental?

De la dimensión actitudinal:

- ¿Cómo valora usted a la educación ambiental?

- ¿Cómo observa el campo de la educación ambiental en los próximos cinco años?

La interpretación de los datos cualitativos, es entendido como ...el proceso mediante el cual se organiza y manipula la información recogida por los investigadores para establecer relaciones, interpretar, extraer significados y conclusiones (Spradley, 1980, p. 70). Los resultados se organizaron de acuerdo a la descripción de los momentos de las prácticas, atendiendo a las explicaciones y justificaciones de los comportamientos.

Como criterios de inclusión de los participantes del estudio se consideró que tuvieran experiencia en el campo de la educación ambiental y con una o más publicaciones referidas a este campo en los últimos tres años. 
Los participantes en el estudio son 13 educadores ambientales de distintos estados de la república mexicana: Michoacán, San Luis Potosí, Chihuahua, Coahuila, Estado de México, Oaxaca, Baja California Norte, Veracruz, Cd. de México, Nuevo León y Yucatán.

En la presentación de las características de la población de estudio se anota entre paréntesis la frecuencia.

- La población femenina (10) es la predominante.

- El promedio de edad es de 43 años

- La mayoría (11) tiene estudios de doctorado: Educación Ambiental (1), Ciencias de la Educación (2), Ciencias Ambientales (1), Química (1), Administración y Educación (1), Investigación Educativa (3), Educación (2).

- Una educadora ambiental tiene la maestría en desarrollo rural, con especialidad en educación

- Un educador ambiental tiene la ingeniería en Horticultura

- El promedio de horas que dedican a la semana a la educación ambiental es de 28 horas y 12 horas a las actividades relacionadas con la investigación.

- Un poco menos del tercio de participantes (5) pertenecen a una red o asociación relacionada con la educación ambiental (Red de Educadores Ambientales de Coahuila; Red de Investigadores de Educación para el Desarrollo Sustentable; Joint Task Force on Forest; Red de Patrimonio Biocultural y CoLaboratorio de Oceanografía Social; Academia Nacional de Educación Ambiental).

- El mayor número de educadores ambientales (11) se encuentra laborando para una institución de educación superior.

- El promedio de años que se han dedicado a la educación ambiental es de 9.5 años.

- El lugar de residencia y laboral, corresponde a distintos estados de la República: Baja California Norte (1), Chihuahua (2), Coahuila (1), Nuevo León (1), San Luis Potosí (1), Oaxaca (1), Ciudad de México (1), Estado de México (1), Michoacán (1), Veracruz (1) y Yucatán (2).

Los resultados comprenden respuestas textuales de las entrevistas, para identificar al educador ambiental, se anota entre paréntesis las iniciales que corresponden al Estado de la república donde radican, en los casos de Chihuahua y Yucatán acompañado de un número: Michoacán, Mn; San Luis Potosí, SLP; Chihuahua, Ch1 y Ch2; Coahuila, Ca; Estado de México, EM; Oaxaca, Ox; Baja California Sur, BCS; Veracruz, Vz; Cd. de México, CdM; Nuevo León, NL; Yucatán, Yn1 y Y2. 


\section{RESULTADOS}

Las representaciones sociales, conforman sistemas cognitivos, de códigos, valores, lógicas clasificatorias, principios interpretativos y orientadores de las prácticas, que se define como conciencia colectiva. Por lo que resultó relevante, en primer término, identificar la identidad al educador ambiental, para posteriormente abordar el sentido que le imprimen a su práctica. La organización de la información se presenta de acuerdo a las categorías que emergen del análisis de información.

\subsection{La identidad como educador ambiental}

La identidad puede considerarse como una construcción social, la cual gradualmente ocurre a partir de los vínculos con otras personas, hechos, acciones y objetos; de acuerdo a Taylor (1993), la identidad consiste en una relación dialógica y de validación de la persona con los otros "los otros significantes". La identidad pasa por procesos de construcción y de reconstrucción de significados a partir de la propia experiencia, y se revela a través de un conjunto de representaciones que pueden ser más o menos estables (Monereo, 2010); se da así una adscripción natural para algunas personas hacia el estudio del medio ambiente; para la mayoría de los educadores ambientales participantes en la investigación, inicia desde temprana edad, “...quizás la educación que recibí en casa, me hizo ser consciente de lo que estamos haciendo al medio ambiente y de ahí me enfoque en lo que fue la biología y en la carrera de biología había como terminales de investigación y siempre me fui más hacia la educación ambiental, la contaminación, los residuos sólidos". (Mn); en este testimonio la educadora ambiental engarza las primeras experiencias de vida con el gusto por el estudio de la biología y de forma posterior con la educación ambiental.

También ocurre esta identidad por la experiencias académicas, hasta por las experiencias obtenidas en el posgrado, "por los estudios de doctorado “... (Ch2). O bien por la práctica profesional: "bueno primero comencé fomentando el cuidado al medio ambiente a la naturaleza, en bachilleres empecé a dar los procesos de biología, de química y demás y es ahí donde yo detecte una necesidad, no se puede enseñar ambiente o medio ambiente sin incluir educación ambiental, no podemos enseñar la naturaleza se compone de esto y esto, sin incluir como es la naturaleza como podemos cuidarla, entonces en realidad mucho tiempo fueron de campaña de difusión de participación activa".. (Ch1). En estas respuestas textuales de las entrevistas se observa el impacto de las experiencias académicas y/o profesionales 
para la adscripción del campo, las relaciones con los docentes y/compañeros, y su influencia significativa en la inclinación o interés por estudiar o trabajar en un campo.

Algunos educadores ambientales aluden a las experiencias formativas desarrolladas con sus alumnos, "...vamos identificando lo que no se debe de hacer, como las acciones irresponsables hacia el ambiente, entonces esa parte para mí ha sido muy importante y estar siempre dispuestos a acompañarnos y eso quiere decir observarnos desde diferentes ojos y desde diferentes caminos" (BCS). En el ejercicio profesional de los educadores ambientales, el contacto con los niños y jóvenes es fundamental para reforzar su identidad con el campo de la educación ambiental.

En ocasiones la incorporación a la educación ambiental ocurre por necesidades institucionales: “...yo comencé que en la educación ambiental porque algún día me dijeron me iba a hacer cargo de una campaña y es cuando realmente yo observé la necesidad de investigar... (NL). También algunos educadores refieren que las exigencias y/o demandas de los sectores en lo que desarrollan sus funciones, los han sensibilizado hacia la importancia de abordar a la educación ambiental.

Otras experiencias significativas corresponden al trato con investigadores $u$ otros educadores ambientales, por ejemplo: “...un investigador de un instituto, el instituto de neurología y básicamente ahí es donde yo me acerco a la investigación, él trabaja a la fecha lo que es la conservación de áreas naturales protegidas, y de las interacciones de los animales (V). Se va creando "escuela", al seguir a otro educador con mayor experiencia.

\subsection{El sentido de las prácticas educativas}

Las representaciones sociales son diferentes en cada grupo, dependen de múltiples factores; en el caso del grupo de educadores ambientales las representaciones obtenidas son homogéneas, de acuerdo a la información que reciben por distintos medios y las comunicaciones que se produzcan van adquiriendo elementos en común.

Las prácticas se concretan en una o en una serie de acciones, es decir la representación que se tiene en la mente de la educación ambiental se manifiesta en el acto, en el hacer. En los resultados se observan tres momentos, definición de las finalidades, planeación de de la práctica y su implementación. Se valoran los alcances de acciones a partir de los posibles resultados, se plantean las acciones con determinadas estrategias y recursos. 
A lo largo de la investigación realizada, los educadores confieren el sentido de las prácticas educativas como formación de conocimientos, actitudes y/valores, procesos de concientización, cambios en los comportamientos. "La educación ambiental es un proceso que permite la toma de conciencia sobre el valor del medio ambiente" (SLP); "...es un proceso que propicia la construcción de conocimiento de las relaciones socioambientales considerando el espacio- tiempo sociohistórico donde se dan dichas relaciones; su finalidad es concientizar sobre la problemática ambiental y encontrar soluciones sustentables a dicha problemática” (Ca); “...trabajando hacia la concientización en el manejo de residuos sólidos y en el manejo del cuidado del agua, podríamos trabajar cuestiones de formación en cuanto a programas o proyectos que tuvieran que ver con el cuidado de la fauna, la flora(Y2); "...se desarrollan saberes y conocimientos referentes al cuidado y preservación del medio ambiente, de ahí que pretende crear conciencia a las nuevas generaciones sobre su importancia y trascendencia a fin de que integren en su actuar cotidiano cierta reflexión para la utilización y consumo" (EM). También se plantea como "Un proceso de enseñanza-aprendizaje de conocimientos y actitudes orientados a mostrar el funcionamiento de los ambientes naturales y cómo los humanos podemos respetar y coexistir de un modo sustentable" (Mn). Estas finalidades coinciden con los resultados obtenidos por Marques, de Cássia y Marques (2019) considerados como propios de una educación ambiental tradicional y conservadora. Sin embargo, también los educadores ambientales en este estudio, consideran a la concientización como la principal finalidad de las prácticas educativas en educación ambiental; para ellos las prácticas se delimitan en un actuar reflexivo y consciente ante los diversos problemas del medio ambiente. Las prácticas educativas se dirigen hacia "... la formación de valores, actitudes, acciones y técnicas a favor del medio ambiente" (Ch1). El componente valoral para los educadores es fundamental, no solo es el complemento del componente cognitivo, se identifica el predominio de una educación ambiental integral (García, 2002).

Los educadores vinculan las prácticas educativas en relación a la existencia de problemas ambientales emergentes en las comunidades: “...entonces empezaron a meter insecticidas, pesticidas y a contratar mucha gente, la gente que contrataron como peones, empezaron a ser productores y se empezaron a llenar aquí que era un lugar de frutos y legumbres, se empezaron a ver flores, pesticidas, insecticidas etc. Entonces se observó el aumento de la contaminación, antes nos bañábamos en cualquier río íbamos a nadar y todo y ahora ya no" ...existe un riesgo en los niños, en esta comunidad han nacido más niños con problemas 
físicos, se planteó la necesidad de educar" (EM). Esta situación expresada por un educador ambiental, refleja la necesidad de educar, para buscar alternativas viables a los problemas ambientales existentes en la comunidad, como informar y dar a conocer el derecho a un ambiente sano.

A partir del conocimiento de las representaciones sociales de los actores educativos, de las singularidades de los conocimientos de sentido común, es posible, develar las relaciones entre las representaciones y las prácticas, en un espacio institucional, social o cultural.

Sentidos de las prácticas. Los educadores ambientales, tienen claro los objetivos de la educación ambiental y el sentido de la práctica educativa. Se identifican principalmente tres sentidos de las prácticas, "desafiantes", "problematizadoras" y "sistémicas".

Las prácticas "desafiantes" dan la posibilidad de "...acompañar a los estudiantes en el proceso educativo que les permita descubrir diferentes problemas medioambientales y sus causas y consecuencias" (Cd. Mx). Se les invita a los estudiantes a cuestionarse el contexto en que viven (Mitra, 2013). O se crean situaciones, para provocar el planteamiento de preguntas de los estudiantes. , por ejemplo: “...el Sendero Educativo, son cuatro jardines conectados por un sendero y cada jardín tiene como una temática específica, uno es el de las plantas medicinales, luego ya metí yo en el siguiente jardín un huerto, y en el siguiente metí un vivero para propagación, y en el otro como una serie de ecotecnias demostrativas" (Ox). Se hace una planeación, con base a retos para los estudiantes, provocando la búsqueda de respuestas.

Las prácticas "problematizadoras", dan la posibilidad de "Acompañar a los estudiantes en el proceso educativo que les permita descubrir diferentes problemas medioambientales y sus causas y consecuencias" (BCS). Los educadores ambientales parten del reconocimiento de la existencia de los problemas ambientales y la necesidad de actuar ante ellos para incidir en la formación de los niños, jóvenes y adultos de conocimientos, actitudes y/o valores. Las prácticas educativas parten del reconocimiento de una necesidad de atender desde una mirada crítica los problemas ambientales. Se "Provoca preguntas, diálogo y abriéndose a lo nuevo" (Da Costa, 2009, p. 191).

Se reflexiona sobre los hechos y procesos observados, para propiciar la desconstrucción, replanteamiento de la situación y reconstrucción -aprehensión del conocimiento. 
La prácticas "sistémicas" promueven la integración de los conocimientos con los saberes, ampliando la mirada de análisis y actuación. "Es la educación a favor del ambiente dirigida a la identificación y resolución de situaciones que afectan el ambiente desde una perspectiva global" (Mn). La mirada de los educadores participantes es amplia, al significar las interrelaciones de las acciones locales, regionales y globales para incidir en un cambio de mentalidad en torno a las relaciones del ser humano con el medio ambiente. Las prácticas educativas “...favorecen el desarrollo de actitudes para proteger o mejorar las relaciones entre el hombre y el medio que lo rodea" (Y2). "Las acciones se dirigen a la identificación y resolución de situaciones que afectan el ambiente desde una perspectiva global" (NL). Este sentido es congruente con la propuesta de Giordan y Souchon (1995), "los estudiantes ante todo, deben saber identificar, jerarquizar y articular imperativos de orden político, económico, social y ecológico" (Giordan \& Souchon, 1995, p. 111).

Estos sentidos de las prácticas educativas, permiten inferir que los educadores ambientales conocen los alcances de la educación ambiental, así como del papel que juegan los diversos actores sociales y las políticas ambientales.

\section{CONCLUSIONES}

La investigación desarrollada tuvo una orientación cualitativa, congruente con el enfoque procesual de las representaciones sociales, con lo cual se prioriza el reconocimiento del sentir y pensar de las personas, con lo cual se logró una configuración inicial de la identidad de los educadores ambientales y del sentido que le imprimen a sus prácticas.

La identidad como educador ambiental. Los educadores ambientales se inscriben al campo de la educación ambiental, porque se identifican con las finalidades y acciones de esta educación, y comparten alguna experiencia significativa en edades tempranas, relacionadas con el cuidado del medio ambiente. En este marco, se observa la importancia de la educación inicial para el desarrollo de actitudes y valores favorables hacia el medio ambiente. Los educadores ambientales desarrollan diversas prácticas, a través de iniciativas propias para hacer más significativos los aprendizajes de sus estudiantes.

El sentido de las prácticas educativas. Los educadores ambientales, construyen prácticas educativas, desarrollando estrategias y acciones pedagógicas fundamentadas en el estudio de las relaciones entre la educación y medio ambiente; le confieren diversos sentidos a las prácticas educativas "desafiantes", "problematizadoras" y "sistémicas", comparten elementos 
en común como los procesos para la concientización sobre los problemas ambientales y la conformación de conocimientos, actitudes y valores. Los educadores ambientales cuentan con información respecto a los procesos educativos, conocimientos, actitudes, saberes y valores, medio ambiente, sociedad, procesos de concientización, relaciones sociedad-medio ambiente, comportamientos y prevención a los problemas ambientales.

Estos resultados confirman porque la teoría de la representación social es cada vez más utilizada en la comprensión de los conocimientos de sentido común que poseen los diversos actores educativos; contribuyen a interpretar el sentido de diversas prácticas, como las referidas a la educación ambiental.

Agradecimientos. La investigación conto con el apoyo del Área Académica de Diversidad e Interculturalidad de la Universidad Pedagógica Nacional, México.

\section{REFERENCIAS}

Calixto-Flores, R., García-Ruiz, M., \& Terrón-Amigón, E. (2015). Experiencias Exitosas en Educación Ambiental. México: UPN. Disponible en http://editorial.upnvirtual.edu.mx/index.php/publicaciones/colecciones/horizontes-educativos/110experiencias-exitosas-en-educacion-ambiental.

Calixto-Flores, R., \& Moreno-Elizalde, L. (2017). Educación ambiental en las instituciones de educación superior. México: ReDIE. Disponible en http://www.redie.mx/librosyrevistas/libros/educamb.pdf

Calixto-Flores, R. (2018). Investigaciones y prácticas pedagógicas en educación ambiental. México: IPEP. Disponible https://www.academia.edu/36106007/INVESTIGACIONES_Y_PRÁCTICAS_PEDAGÓGICAS_EN_EDUCA CIÓN_AMBIENTAL.

Cuevas, Y., \& Mireles, O. (2016). Representaciones sociales en la investigación educativa, Perfiles Educativos, XXXVIII, (153), 65-83. Disponible en http://www.scielo.org.mx/scielo.php?script=sci_abstract\&pid=S0185$26982016000300065 \&$ Ing=es\&nrm=iso.

Da Costa, G.(2009). Educação ambiental crítica: do socioambientalismo às sociedades sustentáveis. Educação e Pesquisa, São Paulo, 35, (1), 145-163. Disponible en http://www.scielo.br/pdf/ep/v35n1/a10v35n1.pdf.

Díaz, A. (1997). La explicación científica. Una polémica desde la teoría del conocimiento. In Hoyos, C. (Coord.). Epistemología y objeto pedagógico ¿Es la pedagogía una ciencia? (pp. 135-148), México: Plaza y Valdés.

García, E. (2002). Los problemas de la educación ambiental ¿es posible una educación ambiental integradora? Investigación la la escuela, 46, $4-25 \quad$ Disponible $\quad$ en https://revistascientificas.us.es/index.php/IE/article/view/7634/6750.

Giordan, A., \& Souchon (1995). La educación ambiental: guía práctica. Sevilla: Díada Editorial.

Marques, G.C., de Cássia, R., \& Marques, R. (2019). Inserção da Temática Ambiental em um Curso de Licenciatura em Ciências Biológicas: Concepções dos Docentes e Suas Práticas Pedagógicas, Pesquisa em Educação Ambiental, 14, (1), 89-110. DOI: http://dx.doi.org/10.18675/2177-580X.

Mitra, S. (2013). El hueco en la pared: sistemas auto-organizados en la educación. Buenos Aires: Editorial Fedum. 
Monereo, C. (2010). La formación del profesorado: Una pauta para el análisis e intervención a través de incidentes críticos. Revista Iberoamericana de educación, 52, 149-162. Disponible en https://rieoei.org/RIE/article/view/615.

Moscovici, S. (1979). El psicoanálisis, su imagen y su público. Huemal. Buenos Aires.

Sautu, R. (2007). Práctica de la investigación cuantitativa y cualitativa. Articulación entre la teoría, los métodos y las técnicas. Buenos Aires: Lumiere.

Spradley, J.P. (1980). Participant Observation, Nueva York: Rinehart \& Winston.

Taylor, C. (1993). El multiculturalismo y la "política del reconocimiento". México: Fondo de Cultura Económica.

Watson-Gegeo, K. (1982). Notas sobre el curso de Introducción a la Investigación Etnográfica en los Campos Educativos y Comunitarios. Massachusetts, USA: Harvard Graduate School of Education. 